\title{
Charles-Bonnet-Syndrom bei Patienten mit Netzhauterkrankung
}

\section{Schlüsselwörter}

Charles-Bonnet-Syndrom · Klinisch · Psychogene Sehstörungen .

Netzhauterkrankung · Visuelle Halluzinationen

\section{Zusammenfassung}

Ziel: Ziel dieser Studie ist die Untersuchung der Prävalenz und der klinischen Merkmale des Charles-Bonnet-Syndroms (CBS) bei einer Gruppe türkischer Patienten mit verschiedenen Netzhauterkrankungen.

Methoden: 264 Patienten mit einem bestkorrigierten Visus von $\leq 20 / 40 \mathrm{im}$ besseren Auge wurden mit einer standardisierten Frage nach dem Vorliegen von CBS-Symptomen befragt. Bei positiver Antwort wurde ein Fragebogen mündlich erhoben, um mehr über die Beschwerden im Einzelnen zu erfahren.

Ergebnisse: An der Studie nahmen 125 (47,3\%) Frauen und 139 (52,7\%) Männer im mittleren Alter von 72,1 Jahren (Spannweite: 31 90 Jahre) teil. Bei 17 Patienten (6,4\%) wurde ein CBS diagnostiziert. 3 Patienten (17,7\%) hatten nichtkomplexe Halluzinationen, 14 (82,3\%) hatten komplexe Halluzinationen.

Schlussfolgerung: CBS ist bei Patienten mit einer Netzhauterkrankung und beeinträchtigtem Sehvermögen nicht ungewöhnlich. Kliniker, die Patienten mit beeinträchtigtem Sehvermögen behandeln, sollten an CBS denken.

\section{Originalartikel}

\section{The Prevalence and Characteristics of Charles Bonnet Syndrome in Turkish Patients with Retinal Disease}

\author{
Serhad Nalcaci ${ }^{\mathrm{a}} \quad$ Orhan Illim $^{\mathrm{a}} \quad$ Zafer Oztas $^{\mathrm{a}} \quad$ Cezmi Akkin $^{\mathrm{a}} \quad$ Ahmet Acarer $^{\mathrm{b}} \quad$ Filiz Afrashi ${ }^{\mathrm{a}} \quad$ Jale Mentes $^{\mathrm{a}}$ \\ Departments of \\ ${ }^{a}$ Ophthalmology and beurology, Ege University Faculty of Medicine, Izmir, Turkey
}




\section{Hintergrund}

Obwohl Charles Bonnet das nach inm benannte CharlesBonnet-Syndrom (CBS) bereits 1760 beschrieben hat, wird den typischen visuellen Halluzinationen bei erheblich sehbehinderten, aber sonst gesunden Menschen unverändert wenig Aufmerksamkeit gewidmet - die Erkrankung ist unter den behandelnden Ärzten zu wenig bekannt. Es kommt neben Lichtblitzen oder geometrischen Formen auch zu komplexen Halluzinationen, die typischerweise in lebendiger Farbigkeit gesehen werden.

\section{Studienergebnisse}

Die Autoren haben an einer Gruppe von 264 türkischen Patienten mit einem Visus unter 0,5 im besseren Auge anhand eines standardisierten Fragebogens die Häufigkeit des Auftretens entsprechender Symptome sowie die Arten der Wahrnehmungen erfragt.

Der Anteil von 6,4\% der Patienten, die Symptome eines CBS angeben, liegt im unteren Bereich bisheriger Mitteilungen, die meist zwischen 10 und 15\% liegen (0,4-63\%). Da, wie es typisch ist, auch hier allen Patienten klar war, dass es sich um nicht reale Wahrnehmungen handelte, hatten neun der $17 \mathrm{Be}$ troffenen bisher niemandem von den Symptomen berichtet. Dies ist am ehesten durch die Sorge zu erklären, dass eine psychiatrische Erkrankung vermutet werden könnte [1]. Dies steht sicher auch in Zusammenhang mit dem in der Regel höheren Lebensalter der Patienten. Die Halluzinationen dauerten bis zu 10 min und traten 10 Monate bis 11 Jahre nach der Sehverschlechterung auf.

Entsprechend den häufigsten Ursachen für eine Sehbehinderung waren auch hier altersbedingte Makuladegeneration (AMD), diabetische Retinopathie, Glaukom und Katarakt die wesentlichen vorliegenden Diagnosen. Letztlich spielt die Ursache der Sehbehinderung wohl keine entscheidende Rolle bei der Entstehung dieser Wahrnehmungen. Allerdings haben Ffytche und Howard im Rahmen einer umfangreichen Untersuchung [2] festgestellt, dass auch bei zerebralen Schäden gleichartige, vor allem strukturelle Halluzinationen entstehen können, die sich nicht von denjenigen unterscheiden, die im Falle einer okulär bedingten Sehbehinderung eintreten. Dies stützt die Erklärung, dass sie als Folge einer Afferenzstörung bzw. eines Wegfalls von sensorischen Reizen auftreten. Dadurch können überlagerte intrazerebrale Muster bewusst werden.

\section{Fazit für die Praxis}

Die Autoren haben mit dieser Studie auf die Möglichkeit eines Auftretens von Halluzinationen hingewiesen, die selbst bei gesunden Menschen auftreten können, aber mit stärkerer Sehbehinderung eine zunehmende Inzidenz haben. Entsprechend der Häufigkeitsverteilung der Sehbehinderung sind vor allem ältere Menschen betroffen und hierbei häufiger Frauen als Männer. Da viele Patienten nicht selbstständig über diese Wahrnehmungsstörungen berichten, sind auch Augenärzte gefordert, hier nachzufragen. Nur dann kann der Patient über den harmlosen Charakter der Halluzinationen aufgeklärt werden, die nicht auf eine psychiatrische Erkrankung hindeuten. Therapeutische Optionen bei stark störendem Charakter sind meist begrenzt, eine Verbesserung des Sehvermögens ist bei den wesentlichen Erkrankungen höchstens bei der Operation einer Katarakt denkbar. Daneben wurden Antipsychotika wie Haloperidol oder Risperidon eingesetzt, auch Antiepileptika haben gute Ergebnisse gezeigt [3]. Nicht selten ist allein durch Lidschluss oder durch bestimmte Augenbewegungen ein Abbruch der Symptome zu erreichen. Kontrollierte Studien fehlen jedoch.

\section{Referenzen}

1 Scott IU, Schein OD, Feuer WJ, Folstein MF: Visual hallucinations in patients with retinal disease. Am J Ophthalmol 2001;131:590-598.

2 Ffytche DH, Howard RJ: The perceptual consequences of visual loss: 'positive' pathologies of vision. Brain 1999;122:1247-1260.

3 Höflich A, Baldinger P, Lanzenberger R, Kasper S, Winkler D: Das CharlesBonnet-Syndrom. J Neurol Neurochir Psychiatr 2012;13:187-189.

Kontaktadresse: Prof. Dr. Klaus Rohrschneider, FEBO, Augenklinik, Universitätsklinikum Heidelberg, Im Neuenheimer Feld 400, 69120 Heidelberg, Deutschland 